Artículo Original

\title{
El poder del diagnóstico clínico en las prácticas de terapeutas ocupacionales que trabajan en contextos educativos
}

\author{
O poder do diagnóstico clínico nas práticas de terapeutas ocupacionais \\ que atuam em contextos educacionais
}

\section{The power of clinical diagnosis in the practices of occupational therapists working in educational contexts}

\author{
Marcela Apablaza Santis ${ }^{a}$ (D), Marichen Castillo Dornemann ${ }^{a}$ (D), Fernanda Daszenies Covacevich ${ }^{a}$ (D), \\ María Isabel Gómez Mansilla ${ }^{a}$ (D), María José Morales Contreras ${ }^{a}$ (D), Daniela Ojeda Aravena ${ }^{a}$ (D) \\ ${ }^{a}$ Universidad Austral de Chile - UACH, Los Ríos, Chile.
}

Cómo citar: Apablaza Santis, M., Castillo Dornemann, M., Daszenies Covacevich, F., Gómez Mansilla, M. I., Morales Contreras, M. J., \& Ojeda Aravena, D. (2021). El poder del diagnóstico clínico en las prácticas de terapeutas ocupacionales que trabajan en contextos educativos. Cadernos Brasileiros de Terapia Ocupacional, 29, e2926. https://doi.org/10.1590/2526-8910.ctoAO2213

\begin{abstract}
$\underline{\text { Resumen }}$
La Terapia Ocupacional es una disciplina emergente dentro de los equipos educativos multidisciplinarios, por lo que, resulta relevante indagar sobre su rol dentro del contexto educativo chileno. Este artículo presenta una investigación cualitativa cuyo objetivo es analizar desde una perspectiva crítica, cómo influye el diagnóstico clínico en las prácticas de terapeutas ocupacionales que trabajan en contextos educativos, considerando como ejes principales para la investigación, el marco normativo inclusivo vigente en Chile, el discurso de terapeutas ocupacionales a cerca de sus prácticas y las relaciones que se establecen con el diagnóstico clínico en este contexto. La muestra corresponde a diez terapeutas ocupacionales que se desempeñan en establecimientos educativos del sur de Chile, a quienes se les aplicaron entrevistas semi-estructuradas. Los resultados permiten visualizar que las políticas educativas influyen significativamente en las prácticas de terapeutas ocupacionales, aún cuando la normativa no especifica el rol disciplinar. Además, el diagnóstico circula fuertemente en el discurso de terapeutas ocupacionales, funcionando como un dispositivo de poder al interior de las prácticas profesionales, especialmente con el fin de validar el rol profesional dentro del contexto educativo. Se discute el uso del diagnóstico en relación a la identidad profesional y los efectos que tiene sobre los sujetos de intervención. El artículo concluye con la propuesta de adoptar una visión crítica del propio quehacer de la
\end{abstract}


disciplina, basándose en la acción transformadora que ésta busca generar a través de la práctica.

Palabras-clave: Inclusión Escolar, Diagnóstico, Políticas Educativas, Discurso, Poder, Terapia Ocupacional.

\section{$\underline{\text { Resumo }}$}

A Terapia Ocupacional é uma profissão emergente nas equipes educacionais multidisciplinares, portanto, é relevante indagar sobre seu papel, no âmbito educacional, em contexto chileno. Este artigo apresenta uma pesquisa qualitativa cujo objetivo foi analisar, a partir de uma perspectiva crítica, como o diagnóstico clínico influencia nas práticas de terapeutas ocupacionais que atuam em contextos educacionais, considerando como eixos principais para a pesquisa, o marco normativo de inclusão vigente no Chile, o discurso dos profissionais sobre suas práticas e as relaçôes estabelecidas com o diagnóstico clínico. A amostra foi composta de dez terapeutas ocupacionais que atuam em estabelecimentos de ensino no sul do Chile, aos quais foram aplicadas entrevistas semiestruturadas. Os resultados demonstraram que as políticas educacionais influenciam significativamente as práticas dos terapeutas ocupacionais, mesmo quando os regulamentos não especificam seu papel disciplinar. Além disso, o diagnóstico circula fortemente no discurso dos terapeutas ocupacionais, atuando como um dispositivo de poder nas práticas profissionais, com o objetivo de validar o papel profissional no contexto educacional. Discute-se sobre o uso do diagnóstico em relaçáo à identidade profissional e seus efeitos nos sujeitos da intervenção. $\mathrm{O}$ artigo conclui com a proposta de adotar uma visáo crítica do próprio papel do terapeuta ocupacional, a partir da ação transformadora que esta profissional busca gerar através da prática.

Palavras-chave: Inclusão Escolar, Diagnóstico, Políticas Educacionais, Discurso, Poder, Terapia Ocupacional.

\section{$\underline{\text { Abstract }}$}

Occupational Therapy is an emerging discipline within multidisciplinary educational teams, therefore, it's relevant to inquire about its role in the Chilean educational context. This article presents research whose aim was to analyze, from a critical perspective, how clinical diagnosis influences the practices of occupational therapists that work in the school system, considering as the main axes for research, the normative framework that governs the educational system in Chile, the discourse of occupational therapists on their practices and the relationships established with the diagnosis in this context. The sample was ten occupational therapists who work in schools from the south of Chile, to whom semi-structured interviews were applied. The results allow us to visualize that educational policies significantly influence the practices of occupational therapists, even when the regulations do not specify the disciplinary role. In addition, clinical diagnosis emerges strongly in the discourse of occupational therapists, functioning as a device of power within professional practices, especially to validate the professional role within the educational context. The use of diagnosis concerning the professional identity and the effects it has on the people they work with is discussed. This article 
concludes with the proposal of adopting a critical vision of the discipline's task, based on the transformative action that it seeks to generate through practice.

Keywords: School Inclusion, Diagnosis, Educational Policies, Discourse, Power, Occupational Therapy.

\section{Introducción}

El sistema educativo chileno, mediante la Ley General de Educación y la Ley de Inclusión Escolar, busca garantizar los principios de equidad, inclusión y calidad en los servicios educativos implementados (Chile, 2009, 2015a). Para dar cumplimiento a los objetivos propuestos y en concordancia con las directrices de organismos internacionales como, el Banco Mundial y la Organización de las Naciones Unidas para la Educación, la Ciencia y la Cultura (UNESCO) (Rizvi \& Lingard, 2009), en las últimas décadas Chile ha implementado una serie de políticas afirmativas orientadas a favorecer la participación de grupos históricamente desaventajados.

Una de las principales estrategias, es el Programa de Integración Escolar (en adelante PIE), cuyo fin es otorgar apoyos educativos específicos para estudiantes tipificados bajo la categoría de Necesidades Educativas Especiales (en adelante NEE) (Chile, 2010a, 2020).

Actualmente, el PIE es considerado uno de los principales recursos de apoyo financiero, destinado específicamente a las escuelas que reciben subvención estatal, pues su funcionamiento, opera mediante el importe de una subvención extraordinaria dirigida a los estudiantes con NEE. De esta forma, las escuelas incorporan en sus equipos técnicos, a profesionales de la salud, tales como psicólogos, educadores diferenciales, fonoaudiólogos y terapeutas ocupacionales (en adelante TTOO), con el fin de suplir los apoyos educativos específicos que requiera su estudiantado (Chile, 2013).

Ahora bien, el acceso al PIE, y por lo tanto a la subvención del Estado, está regulado por los requisitos que establece el Decreto $\mathrm{N}^{\circ} 170$ (Chile, 2010b), siendo el diagnóstico clínico la piedra angular.

La interrelación entre diagnóstico y NEE se enmarca en las dimensiones que establece la Clasificación Internacional del Funcionamiento de la Discapacidad y de Salud (CIF). De esta forma, la determinación de NEE se elabora a partir de la correlación entre el tipo y grado de déficit, el funcionamiento del estudiante respecto al componente físico, a las actividades que es capaz de desarrollar y a las posibilidades de participación efectiva en el medio escolar; así como los factores contextuales ambientales y personales que interactúan con el estudiante (Chile, 2010b).

En el año 2015 se implementa el Decreto $N^{\circ} 83$ (Chile, 2015b), el cual esclarece los criterios y orientaciones para las adecuaciones curriculares, garantizando la flexibilidad de éstas y propiciando la implementación de estrategias inclusivas efectivas, que favorezcan el acceso y progresión de estudiantes con NEE, sobre su currículo.

Finalmente, el 2015, se promulga la Ley de Inclusión Escolar (Chile, 2015a), la cual elimina las barreras de acceso al sistema educativo chileno, a través de tres principios; fin al financiamiento compartido entre el Estado y las familias (elimina el copago de las familias), prohibición del lucro en los establecimientos que reciben aportes estatales y fin a la selección arbitraria de los estudiantes. Sobre este último punto, se prohíbe 
cualquier tipo de mecanismo de selección que condicione la matrícula de estudiantes, tales como selección por rendimiento escolar, pruebas de desempeńo académico, entrevista a las familias, evaluaciones de capacidades y habilidades, diagnósticos, condiciones de salud, entre otros.

Pese a la mirada sistémica que intenta propiciar la normativa durante las últimas reformas, se mantiene que la entrega de recursos, está condicionada al cumplimiento de criterios diagnósticos, basados en el CIE-10 (Clasificación Internacional de Enfermedades) y en el DSM-V (Manual Diagnóstico y Estadístico de los Trastornos Mentales). Asimismo, la normativa determina el tipo de profesional que obtendrá la autorización para evaluar y diagnosticar las NEE de forma diferenciada (Chile, 2010b), generando en la práctica una mirada reducida al diagnóstico biomédico.

Centrar las políticas de inclusión en criterios biomédicos, genera innumerables efectos que han sido ampliamente documentados en la literatura científica y señalados como una de las causas principales, que explican la intensificación de los diagnósticos en contextos escolares, por sobre los procesos de participación educativa y social (Baquero, 2001; Yuing, 2013; Infante et al., 2011).

Lo anterior es nombrado por Baker (2002) como la "verborrea de los acrónimos", refiriéndose a la sobreutilización de siglas asociadas al diagnóstico biomédico. En el caso chileno y de acuerdo a la normativa del PIE, las escuelas han elaborado una prolífica jerga técnica asociada a las NEE: TEA (por trastorno del espectro autista), FIL (por funcionamiento intelectual limítrofe), DIL (por discapacidad intelectual leve), TEL (por trastorno específico del lenguaje), etc. Baker, atribuye dicho efecto a que las escuelas evalúan con mayor frecuencia y a más temprana edad para la obtención de mayores recursos económicos.

Por otra parte, Baker (2002) analiza el efecto del uso del diagnóstico en contextos escolares, concluyendo que su aplicación, basada en criterios de normalidad en función de las capacidades de los estudiantes, conforma una práctica centrada en discursos eugenésicos. Pues, la identificación de estudiantes, su categorización y posterior intervención, bajo la figura del diagnóstico en relación a la discapacidad, configuraría una forma de control de calidad en la población estudiantil. Dicho de otro modo, al construir las escuelas sobre la suposición de un estudiante modelo, basado en un estándar de capacidades y comportamientos, claramente requerirán de servicios especiales para los estudiantes que no responden a criterios de aprendizajes estandarizados, a determinadas capacidades y comportamientos, diferenciándolos del estudiante ideal.

De esta forma, las prácticas clasificatorias han permeado el espacio escolar, donde se genera la etiqueta sobre estudiantes, para así proveer servicios especiales y reconducir a la población escolar hacia fines de gobierno centrados en el desempeño y la productividad. Esto trae como consecuencia ciertas forma de configurar al sujeto estudiante con NEE, bajo parámetros de "capacidad" y de "normalidad", asumiendo a priori, expectativas y pronósticos a cerca de sus procesos educativos (Matus Cánova \& Haye Molina, 2015).

En Chile, este proceso de diagnóstico e intervención está a cargo de equipos multidisciplinarios contratados por las escuelas, en los que participan TTOO. Estos se han incorporado progresivamente al sistema educativo desde hace ya varias décadas, inicialmente a través de las escuelas especiales y posteriormente, a partir del 2000 a través de los PIE (Araya Orellana et al., 2006). Al igual que en otros países, los TTOO que trabajan en contextos escolares, deben enmarcar su quehacer en función de las normativas y leyes establecidas en cada país (Bazyk \& Case-Smith, 2010), siendo el 
abordaje biomédico un elemento axial de la práctica profesional en el caso chileno (Chile, 2013; Apablaza, 2018).

Cabe señalar que en relación a los profesionales competentes para realizar la evaluación diagnóstica, no se considera a los TTOO, sin embargo, las Orientaciones Técnicas para la Implementación del Decreto Nº170 (Chile, 2010b, 2013) incluyen al terapeuta ocupacional como un actor que apoya los procesos educativos inclusivos.

Respecto al rol del terapeuta ocupacional en educación, la WFOT (World Federation of Occupational Therapists) reconoce a la profesión dentro de este campo, indicando que "[...] la terapia ocupacional (en adelante TO) es una profesión que se esfuerza por posibilitar que los individuos alcancen su potencial, desarrollen el significado y el sentido de la vida y aboguen por el logro de las habilidades" (World Federation of Occupational Therapists, 2008). Asimismo, el Colegio de TTOO en Chile y las universidades que imparten la carrera, han reconocido la importancia de la TO en el campo educativo. En un comienzo dicho campo se restringía a las escuelas especiales; luego con la entrada en vigencia de la Ley de Inclusión para las Personas en Situación de Discapacidad (Chile, 2010a) y la creación de los PIE, el colegio de TTOO y las universidades incorporaron el campo educativo como un ámbito profesional (Araya Orellana et al., 2006). Hoy la totalidad de las universidades, en sus planes de estudios, consideran la inclusión escolar como parte de sus ámbitos profesionales, no obstante, en su mayoría incluyen los discursos de inclusión escolar de corriente principal, por sobre la mirada crítica de dichos saberes.

En la literatura científica, la interrelación TO y educación, se ha enfocado en describir la labor y el rol de los TTOO en el contexto escolar desde una perspectiva centrada en la racionalidad técnica. Es decir, en su mayoría las investigaciones se centran en el carácter técnico y funcional del quehacer disciplinar. Específicamente, las investigaciones se han interesado en caracterizar la población a la cual van dirigidas las acciones profesionales (Araya Orellana et al., 2006; Ángulo \& Arenas, 2016), en los fundamentos teóricos que sustentan las prácticas en contextos educativos (Guerra, 2015; Véliz \& Uribe-Echeverría, 2009), en las estrategias de intervención implementadas (Gimeno et al., 2009; Nabiço et al., 2016), en los objetivos propuestos (Cardozo, 2014; Costa da Rosa et al., 2014; Villagra, 2019), en el rol que le compete a la disciplina respecto a las prácticas inclusivas en ambientes escolares (Pires et al., 2018) y en el trabajo colaborativo con los actores del contexto escolar (Villeneuve \& Hutchinson, 2012; Benson et al., 2016).

Si bien esta evidencia constituye un aporte valioso para la disciplina, es necesario profundizar desde un enfoque reflexivo y crítico, respecto a los supuestos que guían estas prácticas y a los efectos que éstas tienen, tanto para los sujetos de intervención, así como también para los contextos escolares; en el entendido que las prácticas responden a regímenes de saber y por tanto constituyen formas de ejercer poder (Foucault, 2002).

Así, desde una perspectiva crítica, recientemente se han desarrollado algunas investigaciones. Prado (2018) analiza desde distintas categorías la TO en educación, haciendo énfasis en la práctica disciplinar, específicamente respecto a ciertas consideraciones y oportunidades para su desarrollo, tanto en Brasil como en el mundo. Entre ellas, señala la importancia de considerar las condiciones político-históricas del país, ya que, desde ahí se proyectan las bases de la institución escolar y es también desde donde se pueden generar las fuentes de exclusión social. Por tanto, realza la importancia de analizar las problemáticas de estudiantes, considerando el contexto y las estructuras 
políticas de forma situada, para así elaborar estrategias de inclusión mediante el fortalecimiento colectivo.

Por otra parte, Apablaza (2018), analiza desde un marco de justicia ocupacional y desde los estudios de gubernamentalidad, las políticas de inclusión chilena. Sus resultados develan por un lado, que la racionalidad de gobierno que subyace a las políticas de inclusión, se centran en el cálculo de los recursos económicos y en la rendición de cuentas. Y por otro lado, expone situaciones de marginación y apartheid ocupacional que evidencian el escaso cuestionamiento sobre los discursos de normalidad y diferencia, que circulan en el contexto educativo.

Por consiguiente y bajo una mirada crítica, esta investigación se centra en rastrear los efectos del diagnóstico clínico sobre las prácticas de los TTOO que se desempeñan en contextos escolares. Específicamente en esta investigación interrogamos las prácticas disciplinares respecto a ¿cómo influye el diagnóstico en las prácticas de TTOO?. De esta forma, problematizaremos la manera en la que los TTOO emplean el diagnóstico, su relevancia en la intervención terapéutica y las formas en las que éste circula dentro de los contextos escolares.

La importancia de explorar la influencia del diagnóstico en las prácticas disciplinares, se relaciona con adoptar una visión crítica del propio quehacer, ya que, si pensamos en la TO como una disciplina que busca generar una acción transformadora (Whalley Hammell, 2015), debemos ser capaces de problematizar cómo nuestras prácticas producen y reproducen formas de pensar los sujetos de intervención y subsecuentemente, los efectos que estas mismas producen en los diferentes contextos en los que se encuentran inmersos.

Para Foucault (2003), una de las tecnologías más significativas de las ciencias biomédicas es el diagnóstico ${ }^{1}$. Este funciona como categoría de intelección, ya no sólo del cuerpo enfermo, sino que hoy por hoy, de la conducta y, por tanto, constituye regímenes de saber-poder y de subjetivación. De esta forma, el análisis biomédico respecto a la conducta, al comportamiento y al cuerpo humano, responde a un conjunto de reglas o criterios, previamente establecidos como formas de verdad. Así el diagnóstico, se vuelve relevante en el contexto educativo funcionando como tecnología de gobierno (Apablaza, 2017), es decir, como una estrategia de demarcación y de diferenciación de la población estudiantil, bajo criterios de salud y enfermedad, y que apuntan a identificar a la población que se desvía de ciertos parámetros de rendimiento y conducta, para luego intervenirla y reconducirla.

En suma, considerando la relevancia que tiene el diagnóstico clínico en el ámbito escolar chileno y la creciente incorporación de la TO al contexto escolar, nos interesa analizar cómo se manifiestan estas relaciones de poder en el ejercicio profesional de TTOO. Para nuestro propósito, hemos organizado el artículo en tres secciones: inicialmente expondremos el diseño y metodología de investigación; posteriormente, presentaremos los resultados y su discusión, para finalmente concluir con las proyecciones y retos disciplinares que derivan de nuestra investigación.

\footnotetext{
${ }^{1}$ Para efectos de esta investigación, asumimos el diagnóstico bajo la noción de dispositivo, propuesta por Michel Foucault (1992), es decir, como una red heterogénea que comprende discursos, instituciones, sistemas de reglas, saberes, instalaciones arquitectónicas, leyes, lo dicho y lo no dicho (Castro, 2018) y cuya propiedad central, es la relación que se establecen entre sus elementos, más que los elementos en sí, para responder a un acontecimiento o urgencia, es decir, posee una función estratégica. En esta línea, el diagnóstico clínico aplicado al contexto escolar, constituye una red heterogénea compuesta por leyes, decretos, normativas, orientaciones técnicas, sujetos (tanto profesionales como estudiantes y familias), plataformas virtuales, saberes biomédicos y saberes 'psi', certificaciones, instrumentos de evaluación, prácticas profesionales, entre otros elementos.
} 


\section{Metodología}

\section{Diseño de investigación y metodología de análisis}

Esta investigación se enmarca dentro del paradigma cualitativo, cuyos supuestos, de acuerdo a Denzin \& Lincoln (2012), se centran en asumir la realidad como un proceso de construcción social, reconocer la implicancia entre quien investiga y el fenómeno de estudio y valorar el carácter situado del contexto en el que se investiga, factores que en su conjunto influyen en la producción de conocimiento que resulte de nuestra investigación. La importancia de seguir este entramado radica en problematizar ciertos regímenes de saber que dada su estabilidad en el tiempo, han sido asumidos como verdades naturales y estables y, por tanto, sin posibilidad de cuestionar.

Con base en los estudios de gubernamentalidad y la noción de dispositivo propuestos por Michel Foucault, analizamos las racionalidades que sustentan las prácticas, estrategias y mecanismos de conducción que operan como tecnologías de gobierno al interior del contexto escolar. La noción de gubernamentalidad, refiere a la mentalidad de gobierno entendida como una forma específica de conducción (Foucault, 2006). Se trataría de las diversas tácticas y estrategias, que de forma articulada y calculada, instituciones y actores, incluyendo al Estado, buscan dirigir la conducta de los sujetos, hacia un fin previamente establecido (Baez, 2014, p. 6). Esta forma de gobierno, requiere de una racionalidad particular, una lógica basada en el cálculo del modo de conducir hacia ciertos comportamientos, donde su novedad estaría dada por la mentalidad que instala en los individuos, por medio del uso de ciertas lógicas (tecnologías), convirtiéndolos en sujetos de autogobierno (Baez, 2014; Lemke, 2011; Rose, 1990, 1998, 2007). Por tanto, el ejercicio de poder estaría dado por la 'conducción de conductas' ('conduct of conducts') bajo una específica administración de posibilidades (Foucault, 2008; Lemke, 2011).

Por otra parte, la articulación entre poder y subjetivación entendida como un continuo, donde las tecnologías del yo y en nuestro caso, las tecnologías 'psi' donde se sitúa el diagnóstico clínico, se consideran como la nueva herramienta que los gobiernos neoliberales, como el chileno, ponen en acción para garantizar la conducción de los individuos. La noción de tecnología propuesta por Foucault (2008), hace referencia a aquel conjunto de prácticas y artefactos estructurados a partir de una racionalidad práctica y que tienen un objetivo más o menos consciente (Rose, 1996 en Hall \& Du Gay, 2011). Es decir, produce modos de existencias, pues su conducción eficaz estaría dada por la aplicación de ciertas estrategias, elaboradas en base a un sistema de cálculo que orienta su elección (Castro-Gómez, 2010). En específico, "Las tecnologías son ensamblajes híbridos de conocimientos, instrumentos, personas, sistemas de juicio, edificios y espacios, apuntalados en el plan programático por ciertos supuestos previos sobre los seres humanos y por objetivos para ellos" (Rose, 1996 en Hall \& Du Gay, 2011, p. 221).

Asimismo, la noción de dispositivo conforma un conjunto heterogéneo de una red cuya potencia se centra en la naturaleza de las relaciones que se establecen entre los elementos que la conforman, su funcionamiento estratégico y su capacidad de ajuste y respuesta ante un acontecimiento o urgencia, son los elementos analíticos claves que guían el tratamiento del material de investigación elaborado.

En esta línea, el diagnóstico clínico, conforma nuestro dispositivo de estudio, cuya forma de funcionamiento y de circulación genera una singularidad interesante de 
examinar en el contexto escolar y en específico, configura, elabora y despliega, es decir, agencia ciertas prácticas en los TTOO que trabajan en el campo escolar.

\section{Corpus de análisis y participantes}

El corpus de análisis se elaboró en base a la normativa vigente vinculada al diagnóstico en contextos escolares, Decreto ${ }^{\circ} 170$ y Decreto n ${ }^{\circ} 83$ (Chile, 2010b, 2015b) y a la aplicación de entrevistas individuales semiestructuradas, registradas a través de audio grabación y posteriormente transcritas. Dado que nuestro interés era rastrear los usos e importancia del diagnóstico clínico, los tópicos de la entrevista se enfocaron en la trayectoria profesional, la relevancia de la normativa legal en las prácticas, las técnicas y abordajes terapéuticos empleados y la caracterización de los sujetos de intervención.

Los criterios para la selección de los participantes fueron: TTOO con al menos un ańo de experiencia profesional en el ámbito educativo, que trabajen en establecimientos escolares de dependencia municipal o subvencionados por el Estado, ubicados entre la X región de Los Lagos y XIV región de Los Ríos en Chile. Estos criterios se establecieron con el fin de descentralizar la investigación académica y construir saberes desde otros territorios; considerando también que nuestra investigación apunta a profundizar el análisis del fenómeno, por sobre generar resultados generalizables y comparativos.

Para la selección de participantes, se utilizó la metodología de avalancha, que según Salamanca \& Martín-Crespo (2007), consiste en que los informantes recomienden a otros participantes, generando una cadena de contactos entre investigadoras y participantes. Complementariamente, se extendió la invitación en diferentes redes de TTOO del sur, base de datos de egresados de universidades regionales y contacto directo con profesionales.

Finalmente, se entrevistaron a diez TTOO mujeres, quienes se desempeñan en escuelas ubicadas en las ciudades de Valdivia, San José, Río Bueno, Osorno, Puerto Montt y Ancud. Las escuelas en las que trabajan son municipales y particulares subvencionados. Sólo una terapeuta ocupacional trabaja en una escuela rural, mientras que las otras profesionales trabajan en contexto urbano. Respecto a las entrevistadas, estas son egresadas de diferentes universidades y trabajan en los respectivos PIE con una trayectoria profesional de entre uno y siete años.

Todas las participantes accedieron de manera voluntaria a formar parte de esta investigación, tras lectura y firma de consentimiento informado, el cual tuvo como fin informar sobre la investigación y cautelar la confidencialidad y voluntariedad de quienes participan en la investigación. Cabe señalar, que por tratarse de población no vulnerable, de acuerdo a las Leyes 25.585 y 20.120 de Chile, no es necesario someter esta documentación a evaluación de Comité ético científico institucional.

\section{Resultados}

\section{El decreto $\mathrm{N}^{\circ} 170$ : ¿quién gobierna a quién?}

Como señalamos anteriormente, uno de los elementos del dispositivo que posicionan al diagnóstico como un requisito central dentro del contexto educativo es la normativa vigente. Por tanto, rastrear el efecto del diagnóstico clínico en la práctica de los TTOO nos obliga a analizar el vínculo existente entre la normativa y el quehacer de los profesionales. 
En relación a la importancia de las normativas para las entrevistadas, estas manifiestan que en general no es algo que determine sus acciones, puesto que el rol de los TTOO no aparece especificado en la normativa, por lo que, refieren no ver sus prácticas supeditadas a esto, sino más bien, como un espacio de mayor libertad. Lo anterior se puede ver reflejado en la siguiente cita:

\section{[...] el rol de la terapeuta ocupacional no está instaurado, ni determinado en las leyes, entonces tú te haces tu rol (Terapeuta 2).}

En contraposición a esto, la ley, plasmada en el Decreto No170 (Chile, 2010b), cobra un rol preponderante y reiterativo dentro del discurso de las profesionales.

[...] a mi me encantaría que los decretos, incluyan la labor del TO, porque no aparece en ninguna parte, [...] cuando yo recién empecé a trabajar, fue complicado porque yo andaba muy a la deriva, muy perdida, pidiendo ayuda a otros colegas de otros colegios (Terapeuta 3).

Cabe señalar que, dentro de la normativa, se describen todas las acciones que deben cumplir otros profesionales tales como psicólogo, fonoaudiólogo, educador diferencial y médico con sus respectivas especialidades, a excepción del TO. De manera minuciosa se destacan los requisitos o metas que se deben lograr y los instrumentos de evaluación a utilizar. Esto se evidencia en el siguiente extracto del Decreto $\mathrm{N}^{\circ} 170$ (Chile, 2010b):

El presente reglamento regula los requisitos, los instrumentos, las pruebas diagnósticas y el perfil de los y las profesionales competentes que deberán aplicarlas a fin de identificar los alumnos con Necesidades Educativas Especiales y por lo que se podrá impetrar el beneficio de la subvención del Estado para la educación especial.

Por lo tanto, cada profesional mencionado en el Decreto, debe cumplir con ciertos compromisos o metas relacionadas con procesos de evaluación específicos, número de horas de atención según tipo de NEE, cupos de estudiantes con NEE por sala, objetivos de trabajo, asistencia, elaboración de informes, recopilación de documentos y actualización de la plataforma virtual, dentro de periodos establecidos (Chile, 2013). En este sentido, la ley indudablemente tiene la autoridad para guiar las prácticas de los profesionales, en coherencia con lógicas de gestión basadas en el accountability o rendición de cuentas.

Haciendo referencia a las tecnologías que propone Foucault (1992), el decreto gobierna a distancia la conducta de los profesionales, generando que ellos mismos deban constantemente revisar sus prácticas para ver si se ajustan o no a las metas que les solicitan, puesto que además, sus resultados son condicionados a la entrega de recursos económicos y de no cumplir con lo establecido en la normativa, se arriesgan a perder el financiamiento estatal, situación que perjudicaría a los estudiantes diagnosticados, quienes dejarían de percibir el apoyo especializado.

En esta línea, Foucault (2009) señala que la eficacia de las formas de gobierno que caracterizan las sociedades neoliberales -a diferencia de los gobiernos disciplinares- es que apuntan a la subjetividad individual, es decir, al autogobierno, más que al cuerpo en sí. Dicho de otro modo, la potencia de la mentalidad de gobierno, en este caso de los 
Estados neoliberales, está dada por la capacidad de conducir la conducta de los individuos, manteniendo la máxima libertad posible (Foucault, 2006). Lo anterior se evidencia en la relación estrecha que se establece entre la normativa y la práctica de TO. A pesar de que los TTOO no poseen un rol definido en el Decreto No170, se ven envueltos dentro de un equipo de trabajo y un programa que les exige el cumplimiento de metas y que, si bien sus acciones no aparecen descritas, ellos deben ajustar sus prácticas en contribución a los objetivos del equipo. Por ejemplo:

Hay mucha burocracia [...] papeleos, términos legales que uno tiene que cumplir porque hay una subvención, un pago asociado [...] uno se ve entrampada (Terapeuta 10).

Una de las formas en que la mentalidad del gobierno central hace efectiva la manera de conducir hacia ciertos fines las prácticas de los profesionales, es condicionar la entrega de recursos económicos a ciertas acciones que debe implementar cada escuela. En este sentido, el criterio económico se superpone al resguardo del derecho a una educación de calidad para todos los estudiantes, quedando los sujetos de inclusión escolar supeditados al cumplimiento de requisitos burocráticos y de control de recursos. En efecto, la manera de acceder a los recursos estatales es, a través, de la categorización de los estudiantes en base a diagnósticos clínicos que han sido enlistados y descritos en la normativa. Esto se refleja en el siguiente extracto de Ley:

Serán beneficiarios de la subvención de necesidades educativas especiales de carácter transitorio aquellos que, en virtud de un diagnóstico realizado por un profesional competente, en conformidad a las normas de este reglamento, presenten algunos de los siguientes déficit o discapacidades... [continúa enlistando todas las NEE transitorias] (Chile, 2010b).

Esto resulta problemático, a la hora de determinar el tipo de estudiante que será sujeto de inclusión, pues por un lado, el Decreto sólo considera como requisito el diagnóstico clínico, basado en criterios de salud y enfermedad, y por tanto, deja en un espacio de exclusión, a aquellos estudiantes que requieren apoyo especializado en razón a otros factores, como por ejemplo migración. Específicamente, la normativa de inclusión escolar chilena, no considera como NEE la situación de estudiantes extranjeros que no dominan el idioma espańol (estudiantes haitianos, palestinos, chinos, por mencionar algunos), o el impacto que produce el cambio cultural y educativo sobre su desempeño escolar.

Por otro lado, el uso del diagnóstico constituye el elemento final que determina la entrada de un tipo de estudiante al PIE y por tanto el acceso a la prestación de los recursos necesarios para su educación, dejando de lado a otros estudiantes que de igual manera requieren apoyo, como el caso de estudiantes migrantes y los efectos que dicho desarraigo produce en los procesos de adaptación ocupacional, en la relación con sus pares, en el sentido de pertenencia a una nueva cultura, etc. Asimismo, determina de manera centralizada (sin considerar el contexto de cada escuela) quiénes son los profesionales idóneos para otorgar cada diagnóstico a un tipo de estudiante determinado arbitrariamente y bajo criterios establecidos a priori. 
Tal como lo señala Foucault (2002), a través de la ley, el diagnóstico operaría como una práctica discursiva hegemónica que organiza los cuerpos, los ordena, los comanda y los determina; puesto que el estudiante sujeto de inclusión y el profesional mandatado a realizar el diagnóstico, será reducido a una categoría biomédica o 'psi' ${ }^{2}$, ya sea quien porte la etiqueta (como estudiante) o quien ejerza la función de etiquetar (el profesional).

\section{Protagonismo del diagnóstico en la práctica}

El diagnóstico en tanto dispositivo, circula entre las prácticas de los TTOO ocupando diversas posiciones que travisten discursos y entremezclan racionalidades orientadas a una acción transformadora inclusiva y a la vez enfocada en saberes biomédicos categorizantes, que terminan esencializando a los sujetos de inclusión que intentan representar.

En un sentido transformador, las entrevistadas inicialmente declaran que el diagnóstico clínico no tiene relevancia dentro de sus prácticas, ya que no resulta necesario para definir o guiar sus intervenciones, considerándolo como algo meramente burocrático. En efecto, al describir el rol de la TO en contextos escolares desde una mirada general, las profesionales hacen referencia a la inclusión desde una perspectiva amplia y caracterizan las problemáticas por sus efectos sobre la participación ocupacional y no por el diagnóstico.

Para mi el diagnóstico es algo netamente burocrático, porque yo siento que desde nuestra disciplina no es necesario del todo tener un diagnóstico para intervenir [...] pero, si el niño no tiene diagnóstico, no se puede subir a la plataforma [y no ingresa al PIE] (Terapeuta 9).

[...] nuestro foco no está centrado mucho en el diagnóstico, entonces más que enfocarnos o darle mayor vuelta a los tecnicismos propios de la patología, nos centramos más que nada en el desempeño de los estudiantes y sus necesidades (Terapeuta 4).

Ahora bien, al especificar sus líneas de intervención y objetivos de trabajo, es decir, hablar sobre su trabajo cotidiano y ya no desde una perspectiva general, las profesionales travisten el diagnóstico entre discursos, cuyo foco de interés se centra en las necesidades de cada estudiante y las dificultades de desempeño que presentan en el contexto escolar. Por tanto, el discurso cambia, sosteniendo que el diagnóstico sí influye, no obstante lo internalizan como una herramienta que permite establecer lineamientos de intervención y de trabajo, junto con guiar el planteamiento de los objetivos terapéuticos. Visto así, el diagnóstico operaría en las prácticas de TTOO, mediante usos elusivos.

[en referencia al diagnóstico] son ciertas características o un conjunto de características o de indicadores [...]. El diagnóstico no limitaría, sí lo que puede es guiar la intervención,

\footnotetext{
${ }^{2}$ En relación a las prácticas 'psi' tomamos la propuesta de Rose (1998) quien hace referencia a lo 'psi' como el conjunto de saberes producidos por las ciencias psicológicas y psiquiátricas y sus efectos sobre las prácticas que entretejen y sostienen y sobre los procesos de subjetivación que en su entramado producen. Pensar lo educativo en clave 'psi' supone una forma de pensar al sujeto, ya sea estudiantes y sus familias, como un objeto de estudio, diagnóstico e intervención por parte de saberes disciplinares provenientes de las ciencias sociales y médicas, en especial del campo de la psicología y la psiquiatría (Arensburg \& Birth, 2002).
} 
para contextualizar a uno en base a qué necesidades se detectan a groso modo y también una visión a futuro sobre las proyecciones que uno tiene con ese niño, las expectativas que también se pueden generar (Terapeuta 10).

Aun cuando el discurso de las TTOO se centra en la inclusión como eje conductor de su quehacer profesional, el diagnóstico clínico en tanto categoría unitaria y centrada en el déficit, cobra protagonismo de manera transversal, siendo empleado para nombrar estudiantes y establecer criterios de ingreso para el área de TO. En este sentido, emplear el diagnóstico como condición para otorgar los servicios de TO en el contexto escolar se fundamenta, en la necesidad de delimitar la población que requiere atención, ante la inexistencia de requisitos establecidos en la normativa vigente. Por tanto, la ausencia disciplinar en la normativa, aun cuando es interpretada como un vacío que otorga mayores posibilidades, la mentalidad de gobierno se hace efectiva, en tanto son las mismas TTOO las que reproducen e internalizan en sus propias prácticas, amarres y sujeciones que la ley no contempla, a diferencia de psicólogos y fonoaudiólogos cuyas prácticas están minuciosamente establecidas y delimitadas en la ley.

[...] en general mi lista de atención son casi puros niños permanentes, son unos cuantos transitorios y dentro de los transitorios están por ejemplo la discapacidad intelectual, los alumnos limitrofes o los déficit atencional (Terapeuta 6).

[...] hay niños TEA [trastorno del espectro austista] que son de alto funcionamiento, que están todos en un curso... que es el curso premiado (Terapeuta 3).

[...] muchos vienen de sectores muy vulnerables, todavía se usan términos, yo misma lo he escuchado, los niños dicen 'por qué estos niños están en esta sala ellos son enfermitos', el vocabulario común 'él es tonto, por eso le cuesta', términos que en el fondo tú los puedes asociar a algún diagnóstico (Terapeuta 7).

Si bien, las participantes reconocen que el diagnóstico estigmatiza, en forma cotidiana y naturalizada lo emplean para nombrar a los sujetos de intervención. Así vemos la forma en la que el diagnóstico cobra protagonismo y actúa en un sentido performativo. Es decir, la circulación recursiva del diagnóstico en el discurso de las TTOO al referirse a sus usuarios, termina fabricando tipos de estudiantes cuya subjetividad queda reducida a la etiqueta diagnóstica.

\section{Los riesgos de una identidad difusa}

La ausencia en la normativa, sumada a una identidad profesional difusa (Turner \& Knight, 2015; Mackey, 2007), genera incomodidad y malestar entre los TTOO que se desempeñan en el contexto escolar. En efecto, la mayoría de las entrevistadas coincide en que al no participar del proceso diagnóstico, ni estipularse el rol de los TTOO en la normativa, son condiciones que se internalizan como una desvalorización de la disciplina, en cuya respuesta se despliegan diversas prácticas para validar su rol frente a los demás profesionales, principalmente ante los docentes. Esta situación, intensificada por el desconocimiento que aún existe entre los equipos profesionales respecto a la 
disciplina y a la propia identidad difusa que ésta posee (Turner \& Knight, 2015), interpela fuertemente la identidad profesional disciplinar, especialmente a la hora de efectuar las intervenciones terapéuticas y vincularse con profesores de aula, ya que, los docentes se muestran reticentes con respecto al trabajo realizado por los TTOO en el contexto educativo, tal como se grafica en las siguientes entrevistadas:

Tiene los dos lados, el lado positivo es que tú puedes moldearte a tú estilo de trabajo, pero el lado negativo es que no entra tu diagnóstico, tus elementos, las características que tú notas, no es algo que esté considerado, y esto le quita validez a la carrera y al profesional (Terapeuta 1).

[En referencia al profesor] El hecho de que al niño lo retire del aula no es que vaya a aprender otra cosa, no es que vaya a desarrollar otras habilidades, sino que le interrumpes su clase y que el niño va a jugar a mi sala y esa es otra dificultad [...] entonces ahi uno tiene que estar validándose (Terapeuta 2).

Dentro de mis diagnósticos, los trabajo yo misma, porque yo igual intento explicarle a los profesores, pero los profes son súper reticentes a lo que uno les dice, si no ando con la bata blanca y con un fonendoscopio yo creo que no pescan (Terapeuta 8).

En respuesta a la interpelación de la identidad profesional, las TTOO recurren a discursos biomédicos y especialmente al diagnóstico, como estrategia de validación frente a los demás profesionales. Este uso práctico del diagnóstico para fines gremiales, resulta efectivo en el contexto escolar, debido por un lado, al estatus de autoridad del cual goza el saber biomédico por sobre el saber pedagógico y por otro lado, debido a la racionalidad objetiva que sostiene el diagnóstico en su categorización de comportamientos que se desajustan de la norma.

Foucault (1992), ya nos anticipaba que el uso del diagnóstico cobra poder en las escuelas en un sentido performativo, es decir, en tanto nombran, identifican y objetivan individuos, para luego intervenirlos y remediarlos. Por tanto, aquellas necesidades escolares que no sean categorizadas bajo la etiqueta diagnóstica, no serán consideradas para la entrega de apoyos. De esta forma, los TTOO, se refugian en el diagnóstico clínico para validar la práctica disciplinar.

Este efecto del diagnóstico sobre la identidad profesional resulta problemática, pues su uso para fines gremiales descentra la atención del sujeto que dice representar, tanto en la política de inclusión, como en los principios rectores de la disciplina. Aun cuando coincidimos que la respuesta desplegada por las TTOO, no son aisladas ni desvinculadas de la tradición histórica de la TO.

\section{Discusión}

En este artículo hemos descrito y analizado el funcionamiento del diagnóstico clínico y sus efectos sobre las prácticas de TTOO que se desempeñan en contextos escolares. Desde la noción de dispositivo, rastreamos y problematizamos las relaciones que el diagnóstico entreteje con la normativa, la función de subjetivación no sólo hacia estudiantes, sino también de los mismos TTOO, los efectos que genera en las formas de 
intervención disciplinar y por último los usos que tiene sobre la identidad profesional, especialmente en relación a la validación del rol profesional.

Un elemento que nos parece interesante analizar y tensionar es la apropiación e internalización del lenguaje biomédico y 'psi' a través del diagnóstico como forma de validación de la identidad profesional. A lo largo del estudio se evidenció que para las TTOO ha sido un gran desafío incorporarse al espacio educativo, tanto por la constante necesidad de validación, como por el continuo desconocimiento del rol que cumple la disciplina y que muy pocos actores educativos logran visualizar como una necesidad. Este imperativo de validación, se entremezcla a su vez con una identidad difusa e híbrida propia de la TO.

En este interjuego, se devela una zona borrosa, poco nítida donde los TTOO desplegamos nuestras prácticas; situación que a nuestro juicio posibilita mayor amplitud de acción que una restricción o limitante del ejercicio profesional. Por un lado, este espacio difuso otorga condiciones de posibilidades con un amplio campo de acción mayor en comparación con otras profesiones, pero que, a su vez, dificulta a otros profesionales del campo educativo, el poder visualizar el rol del TO y su contribución en los equipos de trabajo.

En relación a esto, Clough (2019) en su interés por explorar las decisiones y las formas de intervención que TTOO estadounidenses ponen en práctica en contextos escolares, evidencia que las intervenciones se caracterizan por emplear modelos disciplinares tradicionales, centrados en el desarrollo de destrezas y habilidades, por sobre una práctica centrada en la ocupación.

Por un lado, nuestros hallazgos coinciden en identificar el desconocimiento de otros profesionales sobre el rol del TO, como uno de los factores centrales que impactan en el tipo de intervención que se desarrolla en los contextos educativos. No obstante, nuestros resultados se diferencian en el protagonismo que adquiere el diagnóstico biomédico y 'psi' en la práctica de TTOO en el contexto chileno. Al respecto cabe destacar que una de las diferencias entre la normativa chilena y la estadounidense, es que el rol de TO en esta última, está especificado y fuertemente regulado desde hace varias décadas. Ejemplo de esto son las leyes IDEA (Individual with Disabilities Education Act), ADA (Americans with Disabilities Act,) y el art. 504 de la Ley de Rehabilitación que incluye al TO (Swinth, 2016).

Si bien, podríamos reafirmar que la apropiación y el uso del diagnóstico clínico se debe a la ausencia del rol en la normativa legal, esto pierde fuerza al contrastar nuestros resultados con la investigación de Clough (2019). Pues, independiente que se regule legalmente el rol de TTOO, la necesidad de validación disciplinar se mantiene en ambos contextos. Por tanto, podemos concluir que la necesidad de validación profesional es un factor que influye en los tipos de abordajes y la forma en la que la disciplina se ejerce en contextos educativos, generando por un lado una práctica centrada en el desarrollo de destrezas y habilidades y por otro lado, una práctica centrada en saberes biomédicos y 'psi', ambas alejadas de los marcos contemporáneos disciplinares, donde el foco en la ocupación o en la inclusión es clave.

Por tanto, al contrastar nuestros resultados con la literatura, nos lleva a inferir que la apropiación del diagnóstico clínico en la práctica de TTOO en contextos escolares chilenos, responde más al uso del dispositivo como ejercicio de poder para validarse entre profesionales de diferentes áreas, más que a una fundamentación técnica centrada en los usuarios que dicen representar, lo cual trae consigo efectos que a nuestro juicio son necesarios problematizar. 
Uno de los efectos se relaciona con los sujetos de intervención y las formas de subjetivación que generan decisiones profesionales respecto al empleo del diagnóstico. $\mathrm{Al}$ respecto, Baker (2002) nos advierte que el diagnóstico asume la condición de "enfermedad" en el niño escolarizado, pues la etiqueta, en este caso, vinculada a discapacidad o a NEE, se configura a partir de la ausencia o del déficit de determinadas capacidades requeridas para un desempeño exitoso en el ambiente escolar.

Ligado a la normativa existente, las leyes o decretos no solo estructuran las prácticas, sino que modelan la conducta de lo que se espera y lo que no en un estudiante, razón por la cual los profesionales debemos siempre considerar el poder que el discurso -en tanto prácticas- genera dentro de los espacios de intervención. En relación a esto coincidimos con Baker (2002), quien sostiene que las nuevas nomenclaturas de discapacidad no son solo formas de hablar sobre los nińos y adultos, estas representan lo que Franklin (1994) ha argumentado, como un cambio de la moralización de la discapacidad a la medicalización de la discapacidad.

Por otra parte, Clough (2019) nos alerta sobre el impacto que tiene para nuestra disciplina el emplear un lenguaje biomédico en contextos escolares, advirtiendo que el riesgo es que las otras profesiones reduzcan la disciplina al tratamiento y desarrollo de destrezas y no reconozcan el potencial de nuestra labor sobre la ocupación y los procesos de inclusión.

En este sentido, si bien reconocemos el rol que han posicionado TTOO en los contextos educativos chilenos, creemos necesario afrontar nuevos desafíos y posicionar nuestras prácticas en contextos escolares a partir de marcos de intervención centrados en la ocupación y en la transformación social (Turner \& Knight, 2015; Mackey, 2007; Wilding \& Whiteford, 2008). Por tanto, nos parece necesario distanciarnos de una práctica centrada en saberes que reducen el sujeto a un diagnóstico y más bien ocupar ese intersticio o espacio de fuga, como un campo de acción cuya novedad posibilite ambientes escolares inclusivos.

En suma, relevamos la importancia de asumir responsablemente el uso del discurso clínico y ser crítico en ello, puesto que implica una serie de formas de poder que influyen en los sujetos, pudiendo determinarlos, diferenciarlos, marginalizarlos e incluso llegar a excluirlos. También, nos parece relevante que nuestras prácticas en contextos escolares tensionen el uso del diagnóstico, en tanto tecnología que permite acceder a los servicios profesionales, puesto que a la vez los categoriza y segrega en sus contextos de participación. Por ello, los profesionales debemos ser cuidadosos con el modo en el que nombramos y comprendemos a nuestros sujetos de intervención, para no seguir reproduciendo prácticas y lenguajes que fomenten formas de marginación y exclusión.

Por último, reiteramos las condiciones de posibilidad que tenemos los TTOO en el contexto educativo, pues esa identidad híbrida, confusa y muchas veces incómoda, justamente nos parece un atributo virtuoso para ejercer otras formas de transformación o de resistencia. Por tanto, el llamado es a no quedarnos atrapados en las formas tradicionales de entender al otro, a no reproducir prácticas que reduzcan a estudiantes a una categoría diagnóstica. En palabras de Kumashiro (2000), generar prácticas críticas que transformen no sólo las posibilidades de "otros" sino también desestabilicen las formas opresivas o marginalizantes de ejercer poder. 


\section{Consideraciones Finales}

Con la finalidad de contribuir al campo que abarca la $\mathrm{TO}$ en contextos educativos y descentrar nuestras prácticas del diagnóstico clínico, creemos pertinente referirnos a los alcances de nuestra investigación y delinear también nuevas posibilidades para futuras investigaciones.

Nuestra investigación se centró en los discursos de TTOO. En este sentido, creemos necesario analizar otras materialidades del discurso, tales como observaciones de intervenciones terapéuticas, revisión de instrumentos de evaluación utilizados, registros de intervención, entre otros, los que podrían entregar datos significativos con respecto a los criterios utilizados por los profesionales.

Por otro lado, resulta interesante conocer la percepción de los docentes con respecto a la influencia del diagnóstico clínico en sus prácticas en el aula, visualizando cómo éstas impactan en los procesos de inclusión y cuál puede ser la contribución de los TTOO en este ámbito.

Dicho lo anterior, queda entonces una pregunta abierta a esta investigación. Si la TO estuviera validada dentro del espacio escolar, con un rol definido y con la aprobación de los profesionales, ¿se esperaría un actuar desde lo ocupacional y pedagógico, desligándose de lo clínico o siempre primará este discurso para los profesionales, como una forma de entender a sus sujetos de intervención?

Desde una perspectiva crítica, proponemos reorientar nuestras prácticas hacia las bases filosóficas que fundamentan nuestra disciplina. En concreto proponemos, centrar las acciones de los TTOO que trabajan en contextos escolares, en propiciar y potenciar espacios escolares más democráticos, basados en la perspectiva de derechos y en la participación ocupacional. Para ello, se sugiere descentrar las intervenciones sobre el sujeto individual con NEE y sus habilidades, y generar acciones sobre la cultura escolar, mediante facilitación de espacios de reflexión que problematicen y analicen las prácticas de quienes componen la comunidad escolar y empoderar a las familias y estudiantes a generar y ocupar espacios de participación significativa dentro de sus contextos escolares.

Finalmente, se hace un llamado a las universidades a que incorporen dentro de sus planes de estudios, perspectivas críticas en torno a inclusión escolar, puesto que si persiste una formación centrada exclusivamente en enfoques biomédicos, escasas posibilidades hay de que los futuros TTOO desarrollen prácticas que favorezcan procesos reales de inclusión escolar.

\section{Referencias}

Ángulo, D., \& Arenas, M. (2016). Dificultades de escritura en el contexto escolar chileno: abordaje de terapia ocupacional y la utilización de estrategias de integración sensorial. Revista Chilena de Terapia Ocupacional, 16(1), 57-68. http://dx.doi.org/10.5354/0719-5346.2016.41944.

Apablaza, M. (2017). Prácticas 'Psi' en el espacio escolar: nuevas formas de subjetivación de las diferencias. Psicoperspectivas, 16(3), 52-63. http://dx.doi.org/10.5027/psicoperspectivas-vpl16-issue3-fulltext-1063.

Apablaza, M. (2018). Inclusión escolar, marginación y apartheid ocupacional: análisis de las políticas educativas Chilenas. Journal of Occupational Science, 25(4), 1-13. http://dx.doi.org/10.1080/14427591.2018.1487260.

Araya Orellana, E., Araya Saavedra, C., Caro Vines, P., Espinosa Valderrama, A., González Godoy, C., \& Goudeau Radical, D. (2006). Estudio exploratorio descriptivo de la práctica profesional de los terapeutas ocupacionales en el sistema educativo regular que apoyan los procesos de integración 
educativa en las Regiones Quinta y Metropolitana durante el año 2005. Revista Chilena de Terapia Ocupacional, 6(6), 69-80. http://dx.doi.org/10.5354/0719-5346.2006.113.

Arensburg, S., \& Birth, F. J. (2002). Tecnologías Psi: políticas de la verdad sobre el sujeto/sujeción criminal. Castalia-Revista de Psicología de la Academia, 3(1), 81-93.

Baez, B. (2014). Technologies of government: politics and power in the "information age". USA: Information Age Pubishing.

Baker, B. (2002). The hunt for disability: the new eugenics and the normalization of school children. Teachers College Record, 104(4), 663-703. http://dx.doi.org/10.1111/1467-9620.00175.

Baquero, R. (2001). La educabilidad bajo sospecha. Cuaderno de Pedagogía Rosario, 4(9), 71-85.

Bazyk, S., \& Case-Smith, J. (2010). School-based occupational therapy. In J. Case-Smith \& J. O’Brien (Eds.), Occupational therapy for children (pp. 713-743). Oxford: Elsevier Books.

Benson, J., Szucs, K., \& Mejasic, J. (2016). Teachers' perceptions of the role of occupational. Journal of Occupational Therapy, Schools, \& Early Intervention, 9(3), 290-301. http://dx.doi.org/10.1080/19411243.2016.1183158.

Cardozo, M. (2014). Terapia ocupacional en educación formal, experiencia en el colegio Alemán de CaliColombia. TOG, 11(19), 1-15.

Castro, E. (2018). Diccionario Foucault: temas, conceptos y autores. Buenos Aires: Siglo XXI.

Castro-Gómez, S. (2010). Historia de la gubernamentalidad: razón de Estado, liberalismo y neoliberalismo en Michel Foucault. Bogotá: Siglo del Hombre Editores.

Chile. (2009, 12 de septiembre). Ley n ${ }^{\circ}$ 20370. Establece la Ley General de Educación. Diario Oficial de la República de Chile, Santiago.

Chile. (2010a, 10 de fevereiro). Ley no 20.422. Establece normas sobre igualdad de oportunidades e inclusión social de personas con discapacidad. Diario Oficial de la República de Chile, Santiago.

Chile. (2010b, 21 de abril). Decreto $\mathrm{n}^{\circ} 170$. Fija normas para determinar los alumnos con necesidades educativas especiales que serán beneficiados de las subvenciones para educación especial. Diario Oficial de la Repuiblica de Chile, Santiago.

Chile. Ministerio de Educación de Chile - MINEDUC. (2013). Orientaciones técnicas para programas de integración escolar (PIE). Chile: Ministerio de Educación.

Chile. (2015a, 29 de mayo). Ley n ${ }^{\circ} 20845$. De inclusión escolar que regula la admisión de los y las estudiantes, elimina el financiamiento compartido y prohíbe el lucro en establecimientos educacionales que reciben aportes del Estado. Diario Oficial de la República de Chile, Santiago.

Chile. Ministerio de Educación. (2015b, junho). Decreto no 83. Aprueba criterios y orientaciones de adecuación curricular para estudiantes con necesidades educativas especiales de educación parvularia y educación básica. Diario Oficial de la República de Chile, Santiago.

Chile. Ministerio de Educación de Chile - MINEDUC. (2020). Antecedentes generales PIE. Recuperado el 22 de diciembre de 2020, de https://www.ayudamineduc.cl/ficha/antecedentes-generales-pie-5

Clough, C. (2019). School-based occupational therapists' service delivery decision-making: perspectives on identity and roles. Journal of Occupational Therapy, Schools, \& Early Intervention, 12(1), 51-67. http://dx.doi.org/10.1080/19411243.2018.1512436.

Costa da Rosa, I., Valdivia, B., Cascaes da Silva, F., Barbosa, P., \& Silva, R. (2014). Terapia ocupacional y educación inclusiva: aspectos relacionados al desempeño ocupacional de personas con discapacidad. Revista Chilena de Terapia Ocupacional, 14(1), 123-131. http://dx.doi.org/10.5354/0719-5346.2014.32397.

Denzin, N., \& Lincoln, Y. (2012). Manual de investigación cualitativa: el campo de la investigación cualitativa. Barcelona: Gedisa.

Foucault, M. (1992). Vigilar y castigar: nacimiento de la prisión. Madrid: Siglo XXI.

Foucault, M. (2002). La arqueología del saber. Buenos Aires: Siglo XXI.

Foucault, M. (2003). El nacimiento de la clínica: una arqueología de la mirada médica. Buenos Aires: Siglo XXI. 
Foucault, M. (2006). Seguridad, territorio, población: curso en el College de France:1977-1978. Buenos Aires: Fondo de Cultura Económica.

Foucault, M. (2008). Tecnologías del yo. Buenos Aires: Paidós.

Foucault, M. (2009). Nacimiento de la biopolitica: curso en el College de France (1978-1979). México: Fondo de Cultura Económica.

Franklin, B. (1994). From "backwardness" to "at-risk": childhood learning difficulties and the contradictions of school reform. Albany: State University of New York Press.

Gimeno, H., Pérez, B., Cirez, I., Berrueta, L., \& Barragán, C. (2009). Terapia ocupacional en educación. TOG, 6(Supl. 4), 353-364.

Guerra, G. (2015). Terapia ocupacional en la escuela: de la teoría a la práctica. TOG, 12(7), 115-126.

Hall, S., \& Du Gay, P. (2011). Cuestiones de identidad cultural. Buenos Aires: Amorrortu.

Infante, M., Matus, C., \& Vizcarra, R. (2011). Razonando sobre la idea de diferencia en las políticas educativas chilenas. Universum, 26(2), 143-163. http://dx.doi.org/10.4067/S0718-23762011000200008.

Kumashiro, K. (2000). Toward a theory of anti-oppresive education. Review of Educational Research, 70(1), 25-53. http://dx.doi.org/10.2307/1170593.

Lemke, T. (2011). Foucault, governmentality and critique. Colorado: Paradigm Publishers.

Mackey, H. (2007). 'Do not ask me to remain the same': foucault and the professional identities of occupational therapists. Australian Occupational Therapy Journal, 54(2), 95-102. http://dx.doi.org/10.1111/j.1440-1630.2006.00609.x.

Matus Cánovas, C., \& Haye Molina, A. (2015). Normalidad y diferencia en la escuela: diseño de un proyecto de investigación social desde el dilema político-epistemológico. Estudios Pedagógicos, 41(especial), 135-146. http://dx.doi.org/10.4067/S0718-07052015000300009.

Nabiço, A., Rodrigues, M., \& Costa, S. (2016). Metodologías de intervención de terapeutas del terapeuta ocupacional en contexto escolar con niños con necesidades educativas especiales en Portugal. Cadernos de Terapia Ocupacional da UFSCar, 24(4), 681-693. http://dx.doi.org/10.4322/0104-4931.ctoAO0695.

Pires, S., Moraes, M., Cardoso, P., \& Aparecida, S. (2018). Detalhamento e reflexôes sobre a terapia ocupacional no processo de inclusão escolar. Cadernos Brasileiros de Terapia Ocupacional, 26(2), 381397. http://dx.doi.org/10.4322/2526-8910.ctoAO1203.

Prado, B. (2018). Terapia ocupacional e educação: as proposiçóes de terapeutas ocupacionais na e para a Escola (Tese de doutorado). Universidade Federal de São Carlos, São Carlos.

Rizvi, F., \& Lingard, B. (2009). Globalizing education policy. Londres: Routledge.

Rose, N. (1990). Governing the soul: the shaping of the private self. USA: Taylor \& Francis/Routledge.

Rose, N. (1998). Inventing our selves: psychology, power, and personhood. USA: Cambridge University Press.

Rose, N. (2007). The politics of life itself: biomedicine, power, and subjectivity in the twenty-first century. USA: Princeton University Press.

Salamanca, A., \& Martín-Crespo, C. (2007). El muestreo en la investigación cualitativa. Nure Investigación, 3(27), 1-4.

Swinth, Y. (2016). Educación. In E. B. Crepeau, B. Schell \& E. S. Cohn (Eds.), Willard \& Spackman: terapia ocupacional (pp. 653-657). Madrid: Guanabara Koogan.

Turner, A., \& Knight, J. (2015). A debate on the professional identity of occupational therapists. British Journal of Occupational Therapy, 78(11), 664-673. http://dx.doi.org/10.1177/030802261560143.

Véliz, V., \& Uribe-Echeverría, L. (2009). Aportes de la terapia ocupacional al contexto educacional inclusivo: interrelación entre el enfoque psicosocial, la teoría de integración sensorial y acciones de atención temprana. Revista Chilena de Terapia Ocupacional, 9(9), 103-116. http://dx.doi.org/10.5354/0719-5346.2009.87.

Villagra, A. (2019). ¿Qué de la terapia ocupacional en la escuela? Sesenta años despues. Revista Argentina de Terapia Ocupacional, 5(2), 48-51. 
Villeneuve, M., \& Hutchinson, N. (2012). Enabling outcomes for students with developmental. Qualitative Report, 17(49), 1-29.

Whalley Hammell, K. R. (2015). Client-centred occupational therapy: the importance of critical perspectives. Scandinavian Journal of Occupational Therapy, 22(4), 237-243. http://dx.doi.org/10.3109/11038128.2015.100410.

Wilding, C., \& Whiteford, G. (2008). Language, identity and representation: occupation and occupational therapy in acute settings. Australian Occupational Therapy Journal, 55(3), 180-187. http://dx.doi.org/10.1111/j.1440-1630.2007.00678.x.

World Federation of Occupational Therapists - WFOT. (2008). Declaración de posicionamiento: educación inclusiva en terapia ocupacional. USA: WFOT.

Yuing, T. (2013). De normas y palabras: para pensar la escuela en clave performativa. Praxis \& Saber, 4(7), 103-118. http://dx.doi.org/10.19053/22160159.2051.

\section{Contribución de los Autores}

Esta investigación se enmarca dentro de una investigación de pregrado y forma parte del proyecto FONDECYT 11170479. Marcela Apablaza Santis responsable del Proyecto de Investigación y Profesora Patrocinante del seminario de título. Colaboró en la elaboración del diseño del proyecto, aspectos metodológicos, análisis de los datos y redacción y edición del artículo. Marichen Castillo Dornemann, Fernanda Daszenies Covacevich, María Isabel Gómez Mansilla, María José Morales Contreras, Daniela Ojeda Aravena tesistas del Proyecto de investigación FONDECYT. Cada una colaboró en el diseño del proyecto de investigación de pregrado en todas sus etapas: diseño de la investigación, recolección y análisis de los datos, transcripción de las entrevistas, redacción del artículo y organización de fuentes. Todos los autores aproban la version final del texto.

\section{Fuente de Financiamiento}

Agencia Nacional de Investigación y Desarrollo (ANID),

Proyecto FONDECYT no 11170479. Chile.

\section{Autor para la correspondencia}

Marcela Apablaza Santis

e-mail: marcela.apablaza@uach.cl

\section{Editor de sección}

Profa. Dra. Beatriz Prado Pereira 